\title{
Mixed Medullary-Follicular Carcinoma of the Thyroid: Diagnostic Dilemmas in Fine-Needle Aspiration Cytology
}

\author{
Atef N. Hanna, M.D., Ph.D., Claire W. Michael, M.D., and Xin Jing, M.D. ${ }^{*}$
}

\begin{abstract}
Mixed medullary-follicular carcinoma (MMFC) of thyroid is an extremely rare tumor, characterized by coexistence of morphological and immunohistochemical features of both medullary carcinoma and follicular (or papillary) carcinoma. We herein present fine needle aspiration (FNA) findings of a histology-confirmed MMFC along with a review of literature. The patient was a 64-year-old woman who had a history of Hashimoto's thyroiditis and presented with enlargement of preexisting right thyroid nodule. An US-guided FNA of the thyroid nodule was performed and conventional smears were prepared. A cytologic diagnosis of "positive for malignancy, consistent with medullary thyroid carcinoma (MTC)" was rendered based on the presence of features characteristic for MTC, and the absence of components of follicular neoplasm (adenoma and carcinoma) or papillary carcinoma. However, microscopic examination of the follow-up total thyroidectomy specimen with the aid of immunocytochemical study detected minor portion of follicular carcinoma in addition to MTC. A histologic diagnosis of MMFC was then established. While specific identification of MMFC by FNA may be difficult, it should be emphasized that adequate sampling in conjunction with the proper immunostaining panel could have highlighted the different aspects of the mixed tumor. Diagn. Cytopathol. 2011;39:862-865. @ 2010 Wiley Periodicals, Inc.
\end{abstract}

Key Words: thyroid; mixed medullary-follicular carcinoma; fine-needle aspiration cytology

Unlike papillary thyroid carcinoma, mixed medullary-follicular carcinoma (MMFC) of thyroid is an extremely rare tumor which represents less than $0.15 \%$ of all thyroid tumors. The majority of MMFC occurs in a sporadic form

Department of Pathology, University of Michigan Health System, Ann Arbor, Michigan

*Correspondence to: Xin Jing, M.D., Department of Pathology, University of Michigan Health System, 1500 E. Medical Center Dr., Ann Arbor, MI 48109. E-mail: xinjing@med.umich.edu

Received 29 June 2010; Accepted 2 September 2010

DOI 10.1002/dc.21560

Published online 2 December 2010 in Wiley Online Library (wileyonlinelibrary.com). and the remainder of cases occurs as a component of hereditary syndromes such as multiple endocrine neoplasia (MEN) type 2. Pathologically, the tumor is characterized by coexistence of morphological and immunohistochemical features of both medullary carcinoma and follicular (or papillary) carcinoma. ${ }^{1}$ There are many theories regarding histogenesis of MMFC. As previously reported, mutations in the RET that is similar to or different from those detected in sprodic medullary thyroid carcinoma have been detected in a limited number of cases of MMFC. ${ }^{2-4}$ We herein present fine-needle aspiration (FNA) findings along with histologic features, as well as results of clinical laboratory test including RET mutations in a patient with a histologic diagnosis of MMFC. The challenges associated with the FNA cytologic diagnosis of MMFC will be discussed.

\section{Case Reports}

A 64-year-old woman with a history of Hashimoto's thyroiditis presented with a chief complaint of enlargement of pre-existing right thyroid nodule accompanied by difficulty swallowing. The patient noted a right thyroid nodule and underwent a FNA procedure five years ago. The diagnosis of Hashimoto's thyroiditis was established at that time. Her most recent physical examination revealed that the enlarged nodule measured $2.5 \mathrm{~cm}$ in the greatest dimension. A repeated US-guided FNA was then performed at the outside institution and only conventional smears were prepared during the procedure. The cytological differential diagnoses included neoplasm (Hurthle cell neoplasm versus MTC) and less likely Hashimoto thyroiditis. The patient was then referred to our institution for a definitive diagnosis.

The conventional smears prepared from the FNA of the right thyroid nodule at the outside institution (Fig. 1) revealed moderate cellularity with the cells arranged in a 

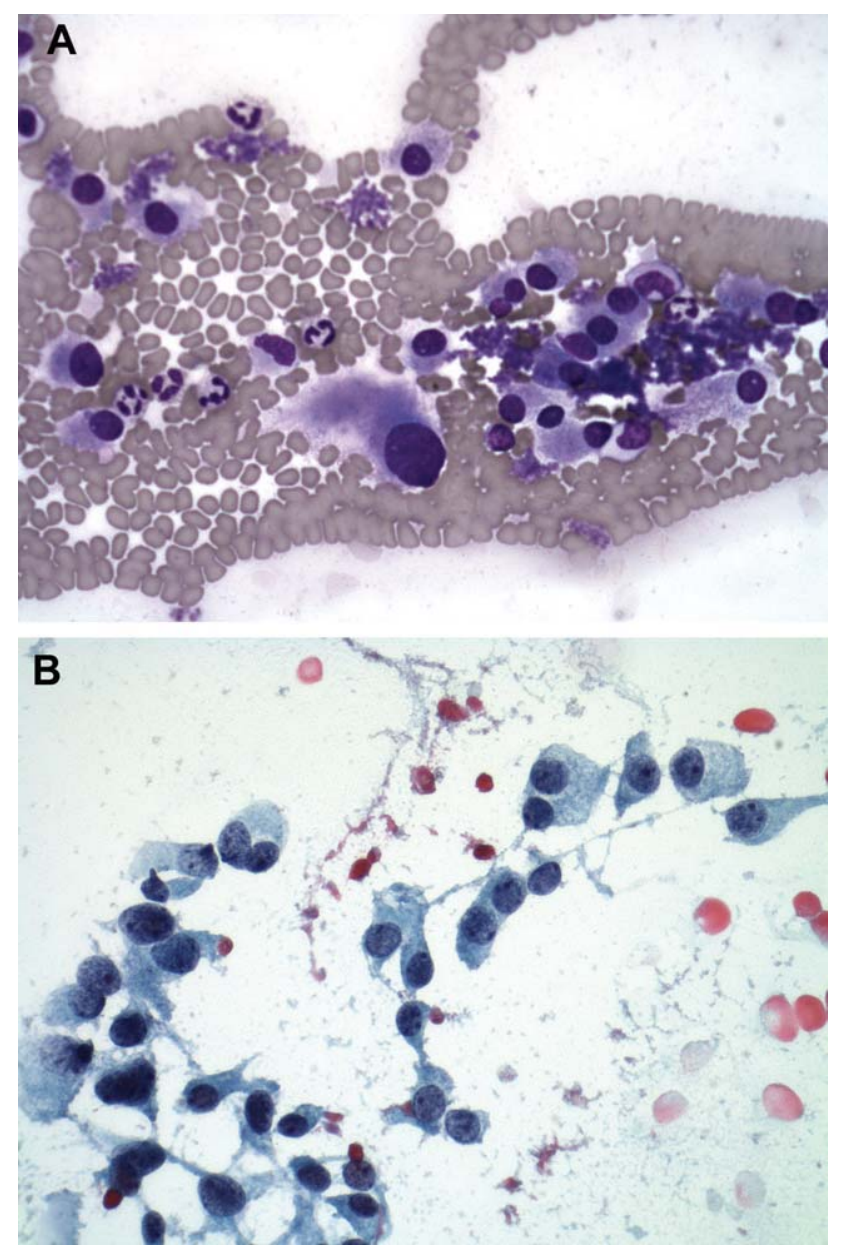

Fig. 1. Conventional smears prepared from the FNA of right thyroid nodule. A: Diff-Quik $\times 200$; B: Papanicolaou $\times 600$. [Color figure can be viewed in the online issue, which is available at wileyonlinelibrary. com.]

dispersed pattern. There are predominantly plasmacytoid cells admixed with minor portion of scattered polygonal cells and spindle cells. The plasmacytoid cells show wellor ill-defined cytoplasmic borders, granular cytoplasm, and eccentrically located nuclei. Occasional bi- or multinucleation and rare giant nuclei are seen. Chromatin has classic salt and pepper pattern. The background is clean without amorphous acellular material (amyloid) identified. Feature of follicular neoplasm or papillary thyroid carcinoma are not seen. Based upon these findings, a cytologic diagnosis of "positive for malignancy, consistent with MTC" was rendered. For further confirmation, the patient's serum level of calcitonin was then tested and the value is $5,741 \mathrm{pg} / \mathrm{ml}$ (normal $<12 \mathrm{pg} / \mathrm{ml}$ ). A total thyroidectomy with central lymph node dissection was subsequently performed. The serum calcitonin level dropped to $7.6 \mathrm{pg} / \mathrm{ml}$ following the surgery.

Gross examination of the surgical specimen revealed a $2.5 \times 1.8 \times 1.5 \mathrm{~cm}$ well-circumscribed mass in the right

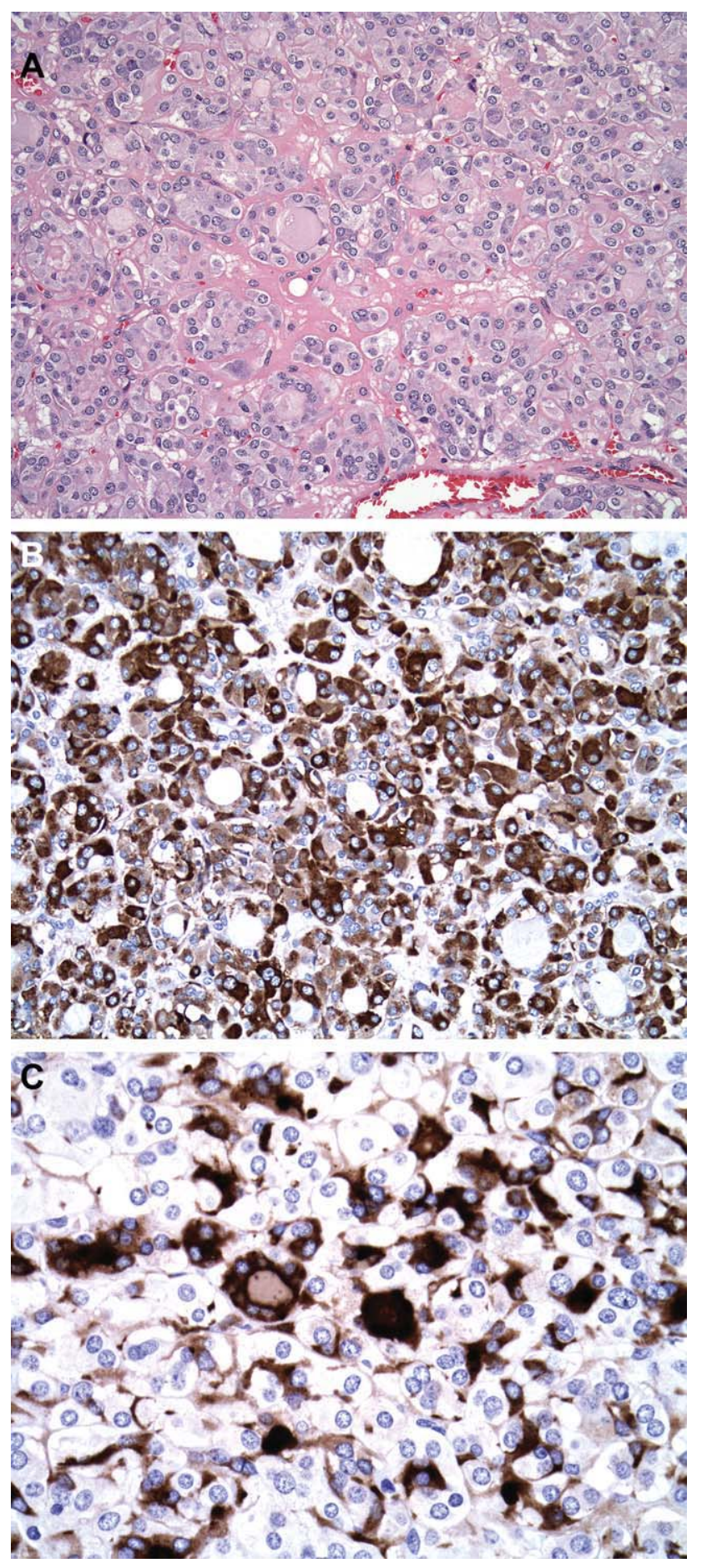

Fig. 2. Histology of follow-up total thyrodectomy and immunostaining profile. A: $H \& E \times 200$; B: calcitonin, $\times 200$; C: thyroglobulin, $\times 200$. [Color figure can be viewed in the online issue, which is available at wileyonlinelibrary.com.]

thyroid. The mass did not invade surrounding capsule. Microscopically, the tumor exhibited predominantly solid growth pattern. In addition, there were tumor cells arranged in follicles that were scattered throughout the 
tumor. Immunostaining revealed that the tumor cells were diffusely positive for calcitonin, CEA, and chromogranin. Further, there are scattered follicles that stained positively with antibody against thyroglobulin (Fig. 2). The histological findings in correlation with the immuonprofile, support a diagnosis of MMFC.

Germline RET proto-oncogene mutation in exons (10, $11,13,14,15$, and 16) was tested using blood sample and mutations were not detected. In addition, results of blood testing for pheochromocytoma (metanephrines) and hyperparathyroidism (intact parathyroid hormone and calcium) were within the normal range. These findings in conjunction with the patient's negative family history and late onset of the symptoms suggest a likelihood of sporadic MMFC.

\section{Discussion}

The patient reported herein was referred to our institution for a definitive diagnosis. The major differential cytologic diagnoses included medullary thyroid carcinoma, Hurthle cell neoplasm and Hashimoto's thyroiditis. The secondary cytologic review revealed features characteric for MTC. These features included but are not limited to dispersed, pleomorphic cells (i.e., plasmacytoid, polygonal, spindle), granular cytoplasm, and classic salt and pepper chromatin. However, it is not uncommon that Hurthle cell neoplasm is mistyped as MTC due to their morphologic resemblance. It has been indicated that the presence of dense cytoplasm and prominent or cherry-red nucleoli favors a diagnosis of Hurthle cell neoplasm. ${ }^{5,6}$ The misinterpretation of MTC as Hashimoto's thyroiditis has also been previously reported. ${ }^{7}$ On the basis of our experience, scant lymphocytes may appear in smears of non-lymphocytic (Hashimoto's) thyroiditis and the lymphocyts may come from peripheral blood during FNA procedure. The lack of moderate to large amount of polymorphous population of lymphocytes in the background, in addition to the absence of follicular/Hurthle cells, makes the diagnosis of Hashimoto's thyroiditis very unlikely. It is noteworthy to mention that MTC may mimic not only any of the primary thyroid neoplasms, but also metastatic neoplasms. Extensive discussion of differential diagnoses of medullary thyroid carcinoma is beyond the scope of this short communication. Briefly, it is infrequent that MTC may exhibit syncytial, follicular or papillary configuration while various portion of dispersed pleomorphic cells may be visualized in follicular neoplasm or papillary thyroid carcinoma; intranuclear inclusions may be seen in both MTC and papillary thyroid carcinoma; Giant, bizarre nuclei seen in anaplastic carcinoma may be occasionally seen in MTC. Further, melanoma, plasmacytoma, spindle cell neoplasm of soft tissue, as well as neoplasms with neuroendocrine differentiation may be mistyped as MTC. ${ }^{8}$ When in doubt, ancillary studies such as serum calcitonin level and/or immunocytohemical (ICC) panel including calcitonin, CEA, chromogranin and thyroglobulin should be used as a vital adjunct in FNA diagnosis of MTC. Most patients have an increased serum calcitonin level of $>10 \mathrm{pg} / \mathrm{ml}$. MTC should stain positive for calcitonin, CEA, and chromogranin; negative for thyroglobulin. ${ }^{9}$ The patient reported herein had a marked elevated serum level of calcitonin prior to the total thyroidectomy and the level dropped into normal range after the surgery. ICC study was not performed at the time of cytologic diagnosis as the cell block was not available.

To our surprise, microscopic examination of the followup total thyroidectomy specimen with the aid of immunohistochemical studies not only confirmed the FNA cytologic diagnosis of MTC but also identified minor portion of tumor cells arranged in follicles that were scattered throughout the tumor and stained positively with antibody against thyroglobulin. A histologic diagnosis of MMFC instead of MTC was thus established. As previously mentioned, MMFC is an extremely rare tumor of thyroid. A few case reports of FNA of MMFC are found in the English literature. Of these cases, none was correctly typed as MMFC by FNA cytologic evaluation. ${ }^{10-13}$ With regard to the case reported herein, we re-reviewed the cytologic material received form the outside institution along with the specimen of total thyroidectomy which was performed in our institution. However, the conventional smears showed MTC only and components of follicular neoplasm were not identified. It appears that sampling issue is the most likely contributor to the mistyping. Almost all MMFC consist predominately of MTC and minor portion of follicular carcinoma that is distributed in a scattered fashion. Obtaining a specimen composed of both MTC and follicular carcinoma components relies on adequate sampling. There is no consensus supporting a minimum number of FNA passes required to obtain adequate samples. In our institution, four to six passes per thyroid nodules has been recommended. ${ }^{14}$ Assuming an ideal cytology sample composed of cells of both MTC and follicular cells was obtained, it is still very difficult using cytomorphologic assessment alone to make a distinction among MMFC, follicular variant of medullary carcinoma and medullary carcinoma with entrapped follicles. ${ }^{15}$ ICC may serve as a valuable adjunct in such a scenario. Thus, additional material should be collected for cell block in an appropriate clinical context that MTC is suspected during the immediate cytologic assessment. It is speculated that a proper ICC panel consisting of antibodies for thyroglobulin, calcitonin, CEA, as well as synaptophysin and/or chromogranin could have highlighted different cell populations.

The majority of MMFC occurs in a sporadic form and rare cases occur as a component of hereditary syndromes such as MEN type 2. Mutations in the RET that is similar to or different from those detected in sprodic medullary 
thyroid carcinoma have been detected in a limited number of cases of MMFC. ${ }^{2-4}$ In our patient, the lack of germline RET proto-oncogene mutation, a negative family history and late onset of the symptoms suggest a likelihood of sporadic MMFC. On the basis of the previously published single case reports, it remains controversial whether MMFC and MTC behave differently in terms of clinical course and management, as well as response to the conventional therapy. ${ }^{11,16,17}$ A possible efficacy of adjuvant radioiodine on the follicular component of MMFC has been suggested though the treatment is mostly surgical intervention which is driven by the medullary component of this mixed tumor. ${ }^{18}$

In summary, while specific identification of MMFC by FNA may be difficult, it should be emphasized that adequate sampling in conjunction with the proper immunostaining panel could have highlighted the different aspects of the mixed tumor.

\section{References}

1. DeLellis RA; International Agency for Research on Cancer; World Health Organization; International Academy of Pathology. Pathology and genetics of tumours of endocrine organs. Lyon: IARC Press; 2004. 320 p.

2. Volante M, Papotti M, Roth J, et al. Mixed medullary-follicular thyroid carcinoma. Molecular evidence for a dual origin of tumor components. Am J Pathol 1999;155:1499-1509.

3. Orlandi F, Chiefari E, Caraci P, et al. RET proto-oncogene mutation in a mixed medullary-follicular thyroid carcinoma. J Endocrinol Invest 2001;24:51-55

4. Frank-Raue K, Dohring J, Scheumann G, et al. New mutations in the RET protooncogene-L881V-associated with medullary thyroid carcinoma and -R770Q- in a patient with mixed medullar/follicular thyroid tumour. Exp Clin Endocrinol Diabetes 2009.

5. Kini SR, Miller JM, Hamburger JI, Smith MJ. Cytopathologic features of medullary carcinoma of the thyroid. Arch Pathol Lab Med 1984;108:156-159.
6. Forrest CH, Frost FA, de Boer WB, Spagnolo DV, Whitaker D, Sterrett BF. Medullary carcinoma of the thyroid: Accuracy of diagnosis of fine-needle aspiration cytology. Cancer 1998;84:295302.

7. Collins BT, Cramer HM, Tabatowski K, Hearn S, Raminhos A, Lampe H. Fine needle aspiration of medullary carcinoma of the thyroid. Cytomorphology, immunocytochemistry and electron microscopy. Acta Cytol 1995;39:920-930.

8. Kini SR. Thyroid cytopathology: An atlas and text. Philadelphia: Wolters Kluwer Health/Lippincott Williams \& Wilkins; 2008. p 276-286.

9. Filie AC, Asa SL, Geisinger KR, et al. Utilization of ancillary studies in thyroid fine needle aspirates: A synopsis of the National Cancer Institute Thyroid Fine Needle Aspiration State of the Science Conference. Diagn Cytopathol 2008;36:438-441.

10. Duskova J, Janotova D, Svobodova E, Novak Z, Tretinik P. Fine needle aspiration biopsy of mixed medullary-follicular thyroid carcinoma. A report of two cases. Acta Cytol 2003;47:71-77.

11. Hsieh MH, Lin MC, Shun CT, Chang TC. Fine needle aspiration cytology of mixed medullary-follicular thyroid carcinoma: A case report. Acta Cytol 2008;52:361-365.

12. Luboshitzky R, Dharan M. Mixed follicular-medullary thyroid carcinoma: A case report. Diagn Cytopathol 2004;30:122-124.

13. Maruna P, Duskova J, Limanova Z, Dvorakova S, Vaclavikova E, Bendlova B. Mixed medullary and follicular cell carcinoma of the thyroid in a 71-year-old man with history of malignant melanoma. Med Sci Monit 2008;14:CS31-CS36.

14. Michael CW, Zhu W. How important is on-site adequacy assessment for thyroid FNA? An evaluation of 883 cases. Diagn Cytopathol 2007;35:183-186.

15. Cakir M, Altunbas H, Balci MK, Karayalcin U, Karpuzoglu G. Medullary thyroid carcinoma, follicular variant. Endocr Pathol 2002;13:75-79.

16. Giove E, Renzulli G, Lorusso C, Merlicco D, Iacobone D. Mixed medullary and follicular carcinoma of the thyroid: Report of one case. Ann Ital Chir 2004;75:251-256; discussion 257.

17. Mizukami Y, Nonomura A, Michigishi T, Noguchi M, Ishizaki T. Mixed medullary-follicular carcinoma of the thyroid gland: A clinicopathologic variant of medullary thyroid carcinoma. Mod Pathol 1996;9:631-635.

18. Nangue C, Bron L, Portmann L, et al. Mixed medullary-papillary carcinoma of the thyroid: Report of a case and review of the literature. Head Neck 2009;31:968-974. 